Acta Crystallographica Section A

Foundations and

Advances

ISSN 2053-2733

Acta Crystallographica Section A FOUNDATIONS AND ADVANCES

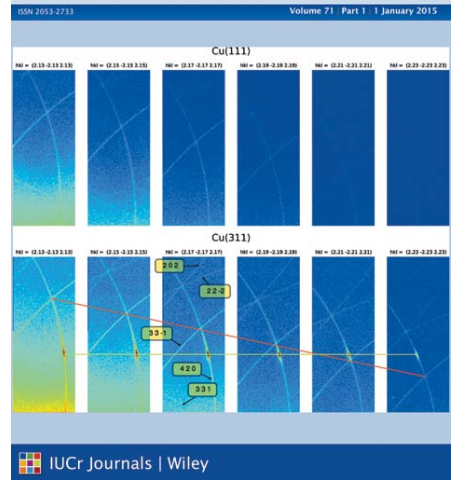

\section{Celebrating the past, looking to the future}

\author{
Simon J. L. Billinge ${ }^{\mathrm{a}, \mathrm{b}_{*}}$ and Jianwei Miao ${ }^{\mathrm{c} *}$
}

${ }^{a}$ Department of Applied Physics and Applied Mathematics, Columbia University, 200 Mudd, 500 W 120th Street, New York, NY 10027, USA, ${ }^{\mathbf{b} C o n d e n s e d ~ M a t t e r ~ P h y s i c s ~ a n d ~ M a t e r i a l s ~ S c i e n c e ~}$ Department, Brookhaven National Laboratory, Upton, NY 11973, USA, and ${ }^{\mathrm{C}}$ Department of Physics and Astronomy and California NanoSystems Institute, University of California, Box 951547, Los Angeles, CA 90095-1547, USA

It is with great excitement that we look forward to the New Year at Acta Crystallographica Section A: Foundations and Advances. As the International Year of Crystallography draws to a close, we were delighted to read in the Nature article The top 100 papers [Van Noorden, R., Maher, B. \& Nuzzo, R. (2014). Nature, 514, 550-553] that crystallography was well represented. We were even more excited to find that a full seven of the top 100 articles were published in IUCr journals, and of these, four were in Acta $A$ (in the order in which they appear in the list):

(1) A short history of SHELX by G. M. Sheldrick [Acta Cryst. (2008). A64, 112-122];

(2) Revised effective ionic radii and systematic studies of interatomic distances in halides and chalcogenides by R. D. Shannon [Acta Cryst. (1976). A32, 751-767];

(3) Phase annealing in SHELX-90: direct methods for larger structures by G. M. Sheldrick [Acta Cryst. (1990). A46, 467-473]; and

(4) Improved methods for building protein models in electron density maps and the location of errors in these models by T. A. Jones, J.-Y. Zou, S. W. Cowan and M. Kjeldgaard [Acta Cryst. (1991). A47, 110-119].

The 2008 paper by George Sheldrick is No. 13 on the top 100 list and is the highest-ranked paper published in the past two decades. As of the end of 2014, it has had over 39000 citations. This speaks to the enduring scientific (and societal) impact of crystallography even into the 21st century, and to the important place that Acta $A$ has in this rich and ongoing history. We are obviously delighted that in this January 2015 edition of Acta $A$ we publish, as one of our three Advances papers, Professor Sheldrick's paper describing SHELXT, a new weapon in the crystallographer's arsenal and a new member of the SHELX suite of programs [Sheldrick, G. M. (2015). Acta Cryst. A71, 3-8]. We believe that, moving forward, the contributions of crystallographers (and nano-crystallographers and non-crystalline crystallographers) will continue to be pivotal and have broad scientific reach, and we believe that Acta A's wonderful tradition and position as the main foundational journal for advances in structure science will be maintained.

We would like to thank our editorial board, reviewers and authors for their hard work in 2014, the more so because striving for the highest standards, as we are, takes more care and application on everyone's part. We strongly believe that the hard work is worth it and that Acta A will go from strength to strength in 2015. The first Advances paper, Magnetic pair distribution function analysis of local magnetic correlations by B. Frandsen, X. Yang and S. J. L. Billinge [Acta Cryst. (2014). A70, 3-11], was published online in December 2013, exactly one year ago. In this January 2015 edition of the journal we have three Advances papers:

SHELXT - Integrated space-group and crystal-structure determination by G. M. Sheldrick [Acta Cryst. (2015). A71, 3-8]; 
Nuclear-weighted X-ray maximum entropy method NXMEM by S. Christensen, N. Bindzus, M. Christensen and B. Brummerstedt Iversen [Acta Cryst. (2015). A71, 9-19]; and

Diffuse multiple scattering by A. G. A. Nisbet, G. Beutier, F. Fabrizi, B. Moser and S. P. Collins [Acta Cryst. (2015). A71, 20-25].

We are also receiving increasing numbers of submissions, for which we thank you. The standards for being published as an Advance are high, and include the broad appeal and potential impact of the work as well as its scientific correctness, but we hope that these papers will be seen as the gold standard for exciting new developments in structure science, which is why we offer accelerated review and production for Advances articles along with promotion after they are published. We encourage you to submit your best work to us for consideration as an Advance.

The Advances section will also be featuring a number of Lead Articles in 2015, in which well known experts will be providing useful overviews of different fields of research. We have several articles in the pipeline, including ones discussing electron crystallography of protein microcrystals, quantitative scanning transmission electron microscopy, the complementary nature of powder diffraction and electron crystallography, and cryogenic coherent X-ray diffraction imaging of biological particles. Suggestions from our readers for topics for Lead Articles are also welcome.

Looking forward, we would like to expand the scope of Acta $A$ and include some new directions, such as X-ray free-electron lasers (XFELs), various coherent diffractive imaging (CDI) methods (such as plane-wave CDI, Bragg CDI and ptychographic CDI), serial crystallography, aberration-corrected electron microscopy, electron and X-ray tomography, nanocrystallography, disorder in materials, dynamics and time-resolved experiments, and so on.

As we strive to keep Acta $A$ at the forefront of developments in crystallography and structure science, we are adopting higher standards for acceptance of articles across the board. For example, beyond just describing a development, each article should identify the rationale for making the development and how it would be used by the crystallographic community, and we look forward to collaborating with our authors to bring this about.

Finally, we would like to wish all of our authors, editors, reviewers and readers a wonderful, prosperous, productive and happy 2015. 